\title{
Effects of noise upon introverts and extroverts
}

\author{
LIONEL STANDING, DANNY LYNN, and KATHERINE MOXNESS \\ Bishop's University, Lennoxville, Quebec, Canada
}

\begin{abstract}
The effects of noise upon arousal, mood, and cognitive performance were examined in two experiments employing selected groups of introverts and extroverts as subjects. In the first experiments, white noise at $60 \mathrm{~dB}$ produced higher physiological activation in introverts, but not in extroverts, as predicted from Eysenck's theory of personality. Likewise, performance in a comprehension task was impaired in introverts but not in extroverts. Extroverts were also shown to display more subjective deactivation under noise, and to have greater habitual tolerance for noisy real-world environments. In the second experiment, exposure to patterned noise increased state anxiety in both introverts and extroverts, but did not affect aggressiveness.
\end{abstract}

Although the effects of noise upon various types of cognitive skills have been widely studied (see, e.g., Holding \& Baker, 1988), and significant performance decrements have been found in the more aversive conditions (typically $80-90 \mathrm{~dB}$ ), there are relatively few studies of the effects of lower level noise upon behavior, and of its interaction with personality types.

Moderate noise may readily be used to produce arousal and a range of behavioral consequences (Eysenck, 1975). According to Eysenck's theory of personality (Eysenck, 1967, chap. 3), introverts typically show less inhibition of the arousal response to external stimuli than do extroverts. This elevated arousal should lead to poorer cognitive performance for introverts under noise, if noise is conceived as producing irrelevant responses. In accord with this prediction, Daoussis and McKelvie (1986) found that introverts' performance on a reading-comprehension test decreased by $33 \%$ when a soft background of rock music was present, whereas extroverts' performance was unaffected. Furthermore, on the basis of survey research, Weinstein (1978) has reported that introverts typically show an aversion to noise in the real world.

The above results suggest that even moderate levels of noise affect mood and performance, and that the personality dimension of introversion-extroversion mediates this process. In the present investigation, we examined whether low-level noise leads to greater psychological and physiological activation, and impaired cognitive performance, among introverts as compared with extroverts, as predicted from Eysenck's theory. We also examined whether introversion is associated with noise sensitivity in general, defined as a habitual preference for quiet surroundings.

The helpful advice of Stuart McKelvie is gratefully acknowledged. These studies were reported in honors theses prepared by the second and third authors, supervised by the first author. Danny Lynn is now at the Law School, University of Alberta, Edmonton. Reprints and the PNS questionnaire may be obtained from L. Standing, Department of Psychology, Bishop's University, Lennoxville, Quebec J1M 1Z7, Canada.

\section{EXPERIMENT 1}

\section{Method}

Subjects. The subjects were 60 undergraduate students ( 30 extroverts and 30 introverts), with a mean age of approximately 21 years.

Stimuli. The quiet and noise conditions involved white noise at 45 and $60 \mathrm{~dB}(\mathrm{~A})$, respectively, as measured on a Simpson 885 sound meter. The noise stimulus was played on a Bell and Howell 3091 tape recorder.

Materials. The reading-comprehension material comprised an academic passage of $\mathbf{4 5 0}$ words, taken from the preparatory booklet for the Law School Admissions Test, describing Wagnerian art in Diaghilev's ballet productions. This material required detailed study for mastery.

Subjective activation was assessed by means of the ActivationDeactivation Adjective Check List, or AD-ACL (Thayer, 1967). Physiological arousal was measured by determining the subject's finger pulse with a Pulsetronix 1000 meter. Extroversion was measured with the Eysenck Personality Inventory (Eysenck \& Eysenck, 1963).

The subject's habitual noise preference was assessed by means of the Preference for Noise Scale (PNS), devised by the present authors. Broadly similar to the noise-sensitivity scale of Weinstein (1978), this questionnaire comprises 15 items, which are answered on a 7-point scale (e.g., "Is a quiet living area important to you?" "Do you prefer music generally to be soft?"). A high score (maximum 105) indicates a strong dislike of noisy real-life situations.

Procedure. An initial pool of 160 randomly selected undergraduates completed the Eysenck Personality Inventory. The extrovert and introvert subject groups ( 30 each) were then selected as the top and bottom scorers, respectively, on the extroversion scale.

Each subject group was randomly divided into two halves, which were then assigned to the quiet and the noise conditions. The noise, which was turned on before any subjects arrived, entered the testing room from an adjacent laboratory, through a door vent; a notice on the door referred to another experiment in progress. The subjects were tested in groups of 4 to 16 . Once seated, they were instructed that they would complete a test of reading comprehension, followed by two questionnaires, and would be given a pulse measurement.

The reading-comprehension task was given as $10 \mathrm{~min}$ of study time, followed by a true-false comprehension test of 12 items. This was followed by the AD-ACL and the PNS questionnaires, appropriate instructions being given orally. Finally, the pulse reading was taken. The entire procedure required $25 \mathrm{~min}$.

The experimental design represented a $2 \times 2$ factorial with independent groups, where Variable A represented the quiet/noise variable and Variable B represented the introvert/extrovert variable.

\section{Results and Discussion}

An independent groups $2 \times 2$ analysis of variance (ANOVA) was performed for each dependent variable. The group means are shown in Table 1. 
Table 1

Mean Pulse Rate, Reading-Comprehension, Activation and Deactivation, and Preference for Noise Scale Scores for Introverts and Extroverts under Quiet and Noise Conditions in Experiment $1(n=15)$

\begin{tabular}{|c|c|c|c|c|c|c|c|c|c|c|}
\hline \multirow{2}{*}{$\begin{array}{c}\text { Personality } \\
\text { Type }\end{array}$} & \multicolumn{2}{|c|}{ Pulse Rate } & \multicolumn{2}{|c|}{$\begin{array}{c}\text { Reading } \\
\text { Comprehension } \\
\end{array}$} & \multicolumn{2}{|c|}{ Activation } & \multicolumn{2}{|c|}{ Deactivation } & \multicolumn{2}{|c|}{$\begin{array}{l}\text { Preference } \\
\text { for Noise }\end{array}$} \\
\hline & $M$ & $S D$ & $M$ & $S D$ & $M$ & $S D$ & $M$ & $S D$ & $M$ & $S D$ \\
\hline \multicolumn{11}{|c|}{ Quiet } \\
\hline Introvert & 72.8 & 8.47 & 11.13 & 1.92 & 2.03 & 0.72 & 2.47 & 0.51 & 4.56 & 0.90 \\
\hline Extrovert & 78.8 & 7.95 & 11.20 & 1.86 & 2.02 & 0.72 & 2.51 & 0.68 & 3.80 & 0.95 \\
\hline \multicolumn{11}{|c|}{ Noise } \\
\hline Introvert & 82.27 & 14.65 & 9.2 & 1.97 & 1.98 & 0.55 & 2.29 & 0.66 & 4.28 & 0.64 \\
\hline Extrovert & 76.40 & 16.15 & 11.0 & 1.69 & 2.01 & 0.64 & 2.89 & 0.49 & 3.54 & 0.80 \\
\hline
\end{tabular}

Pulse. No main effect of extroversion or of noise upon pulse rate was found $[F(1,56)=1.23$ and $F(1,56) \leq 1$, both $p s>.05$ ]. However, a marginally significant interaction between these variables occurred $[F(1,56)=3.5$, $p<.06$ ], such that noise increased the introverts' pulse, whereas the extroverts' declined trivially. Within the quiet condition, the introverts had a lower pulse than did the extroverts $[t(28)=2.0, p<.05]$.

Subjective activation and deactivation. The activation data for the AD-ACL showed no significant trends with noise or extroversion (both $F \mathrm{~s}<1$ ). Furthermore, the deactivation data from the AD-ACL showed no main effect of noise $[F(1,56)=2.67, p>.05]$. However, noise caused the extroverts to become more deactivated than in the quiet condition $[t(28)=1.73, p<.05]$. They were also more deactivated than the introverts under noise $[t(28)$ $=2.85, p<.01]$. These results suggest an arousal decrease among extroverts under noise.

Reading comprehension. The introverts and extroverts performed equally well in the quiet condition. Under noise, the extroverts continued to perform at the same level, but the introverts showed decreased performance $[t(28)=2.72, p<.01]$. This score was also lower than that of the extroverts under noise $[t(28)=2.68, p<.01]$.

Preference for noise scale. The PNS scores were not affected by the noise variable $[F(1,56)=1.57, p>.05]$. However, the introverts scored markedly higher $[F(1,56)$ $=12.23, p<.01]$, indicating a greater habitual dislike of noise. Pooling all subjects, the PNS and extroversion scores were negatively correlated $[r(58)=-.49, p<$ $.01]$.

The present results largely agree with the experimental predictions. They also replicate the findings of Daoussis and McKelvie (1986), in that reading comprehension was significantly inpaired by noise for introverts but not for extroverts, and demonstrate that this effect may be obtained with white noise, rather than rock music, as the noise stimulus.

In the present experiment, the introverts' pulse rates were increased by noise, unlike those of the extroverts, which were unaffected by noise. The extroverts also had a higher deactivation score in noisy as opposed to quiet conditions. In contrast, the introverts' deactivation scores did not change from quiet to noise, and they were less deactivated than the extroverts when both were under the noise condition. Overall, the introverts tended to show increased arousal under noise, whereas the extroverts showed unchanged or decreased arousal.

Under quiet conditions, the introverts showed a lower pulse rate and were as deactivated as the extroverts. Therefore, the problem for introverts appears to be that they exhibit an excessive arousal response to noise, rather than that they are initially too aroused before the noise begins.

The PNS scores, an index of self-rated sensitivity to noise, suggest that introvert subjects have a greater habitual dislike of real-life noise than do extroverts, in accordance with the other data indicating their elevated noise susceptibility. The PNS scores were not affected by whether the questionnaire was filled out in noise or quiet conditions, and presumably represent a subject trait rather than the momentary influence of the testing environment.

\section{EXPERIMENT 2}

Mood changes under noise have not been as widely studied as task performance; however, they can provide a sensitive indication of noise effects. Edsell (1976) found that state anxiety, as measured by the State-Trait Anxiety Inventory (STAI), was raised by exposure to white noise at 61 and $75 \mathrm{~dB}$, relative to a control condition at $51 \mathrm{~dB}$, all conditions involving concurrent social stress. Standing and Stace (1980) replicated Edsell's main findings in a simpler situation without social stress; they used noise levels of 61 and $75 \mathrm{~dB}$, with a control condition of $43 \mathrm{~dB}$. Heightened state anxiety occurred after $30 \mathrm{~min}$ of noise exposure at $75 \mathrm{~dB}$, whereas increased variability of anxiety scores was noted at $61 \mathrm{~dB}$. Furthermore, the results of Smith and Morris (1977) suggest that anxiety is also increased by stimulative music. Subjects exposed to stimulative music, as opposed to sedative music or no music, showed increased worry and impaired concentration; their expectancies of performance were also lower. Noise has also been reported to increase aggressiveness in some situations (Mueller, 1983). 
Table 2

Man State-Anxiety and Aggressiveness Scores for Introverts and Extroverts under Quiet and Noise Conditions in Experiment $2(n=30)$

\begin{tabular}{lrrrrr}
\hline \multirow{2}{*}{$\begin{array}{c}\text { Personality } \\
\text { Type }\end{array}$} & \multicolumn{2}{c}{ State Anxiety } & & \multicolumn{2}{c}{ Aggressiveness } \\
\cline { 2 - 3 } \cline { 5 - 6 } & $M$ & $S D$ & & $M$ & $S D$ \\
\hline & \multicolumn{5}{c}{ Quiet } \\
Introvert & 40.0 & 8.12 & & 17.35 & 8.73 \\
Extrovert & 35.8 & 7.13 & & 17.42 & 5.52 \\
& & Noise & & \\
Introvert & 51.01 & 12.35 & & 23.82 & 7.23 \\
Extrovert & 45.18 & 11.16 & & 19.15 & 5.63 \\
\hline
\end{tabular}

Experiment 2 was planned to study the effects of patterned noise (garbled speech) upon anxiety and aggressiveness, again comparing introverts and extroverts. It was predicted that any noise effects should be enhanced for the introverts.

\section{Method}

Subjects. The subjects were 60 undergraduate students ( 30 extroverts and 30 introverts), with a mean age of approximately 19 years.

Apparatus. The STAI (Spielberger, Gorsuch, \& Lushene, 1970) was used to measure state anxiety. A word-association test, taken from Feshbach (1961), was used to assess aggressiveness. The noise stimulus comprised a tape recording of three superimposed radio talks, played at $70 \mathrm{~dB}(\mathrm{~A})$, whereas the quiet condition involved $45 \mathrm{~dB}$ of background white noise.

Procedure. The subjects were selected from a pool of 160 students, as the $\mathbf{3 0}$ highest and $\mathbf{3 0}$ lowest scorers on the Eysenck Personality Inventory extroversion scale. Each group had 20 females and 10 males. The noise and no-noise groups were formed by randomly assigning 15 introverts and 15 extroverts to each. The subjects were tested in groups of 3. While exposed to the appropriate noise level ( 70 or $45 \mathrm{~dB}$ ), the subjects first completed the STAI questionnaire. They were then presented, visually and orally, with a sequence of six neutral words and five words with aggressive connotations (wash, choke, travel, massacre, walk, murder, relax, stab, sleep, torture, listen). The subjects were asked to write down 10-word associations to each stimulus, presented at 1min intervals.

\section{Results and Discussion}

The STAI scores were analyzed by a $2 \times 2$ ANOVA for independent groups, which indicated that state anxiety was raised by noise $[F(1,52)=12.7, p<.01]$. No effect of extroversion was found, nor any interaction (both $F \mathrm{~s} \approx 1)$. The group means are given in Table 2 . The finding that heightened anxiety is caused by moderate noise replicates the results of Standing and Stace (1980). Since the anxiety measure did not discriminate between introverts and extroverts, it appears that different mechanisms are involved in the anxiety response and the previously observed cognitive performance decrement.

The word-association data were analyzed to determine the number of aggressive associations to each aggres- sive stimulus word, as rated by three independent scorers (interrater reliability $=.89$ ). No effect of either noise or extroversion upon aggressiveness was found (both $F \mathrm{~s} \approx 1)$.

\section{GENERAL DISCUSSION}

The present study emphasizes that even modest levels of noise may be harmful to some individuals within the population, reinforcing Bryant's (1973) argument for recognizing the special needs of noise-sensitive persons. The obtained data show that such individuals are likely to be introverts when cognitive performance, noise preference, or activation is the dependent measure, although noise was found to increase anxiety equally in introverts and in extroverts and did not affect aggressiveness in either group. The typically negative reactions of introverts to noise have previously been noted by Weinstein (1978), and by Stanford, Angelini, and Raphael (1985). There may be other high-risk groups, as yet unstudied.

\section{REFERENCES}

Bryant, M. (1973). Noise laws don't protect the sensitive. New Scientist, 27, 738-740.

Daoussis, L., \& McKelvie, S. (1986). Musical preference and the effects of music on a reading comprehension test for extraverts and introverts. Perceptual \& Motor Skills, 62, 283-289.

EDSELL, R. (1976). Anxiety as a function of environmental noise and social interaction. Journal of Psychology, 92, 219-226.

EYSENCK, H. (1967). The biological basis of personality. Springfield, IL: Thomas.

EYSENCK, H., \& EYSENCK, S. (1963). Manual for the Eysenck personality inventory. San Diego: Educational \& Industrial Testing Service.

EYSENCK, M. (1975). Effects of noise, activation level, and response dominance on retrieval from semantic memory. Journal of Experimental Psychology: Human Learning \& Memory, 104, 143-148.

FESHBACH, S. (1961). Stimulating versus cathartic effects of a vicarious aggressive activity. Journal of Abnormal \& Social Psychology, 63, 381-385.

HoLDING, D., \& BAKER, M. (1988). Toward meaningful noise research. Journal of General Psychology, 114, 395-410.

MUELLER, C. (1983). Environmental stressors and aggressive behavior. In R. Geen \& E. Donnerstein (Eds.), Aggression: Theoretical and empirical reviews (Vol. 2, pp. 51-76). New York: Academic Press.

SMITH, C., \& MoRrIS, L. (1977). Effects of stimulative and sedative music on cognition and emotional components of anxiety. Psychological Reports, 38, 1187-1193.

SPielberger, C., Gorsuch, R., \& LuShene, R. (1970). Manual for the state-trait anxiety inventory. Palo Alto, CA: Consulting Psychologists Press.

StANDiNG, L., \& STACE, G. (1980). Effects of environmental noise on anxiety level. Journal of General Psychology, 103, 263-272.

Stanford, R., ANgelini, R., RAPhael, A. (1985). Cognition and mood during ganzfeld: Effects of extraversion and noise versus silence. Journal of Parapsychology, 49, 165-191.

THA YeR, R. (1967). Measurement of activation through self-report. Psychological Reports, 20, 663-678.

Weinstein, N. (1978). Individual differences in reactions to noise: A longitudinal study in a college dormitory. Journal of Applied Psychology, 63, 458-466.

(Manuscript received July 18, 1989.) 